\title{
Borderline Personality Disorder (BPD): Approach by Dialectic-Behavioral Therapy (DBT)
}

\author{
José Luis Triviño ${ }^{1}$
}

María Ángeles Ortega ${ }^{2}$

${ }^{1}$ UNED Faculty of Science. Barcelona, Spain, ${ }^{2}$ UB Faculty of Psychology. Barcelona, Spain

Introduction. Borderline Personality Disorder (BPD) is arguably the most common subtype of Personality Disorder seen by services (Coid et al, 2006; de Ruiter \& Greeven, 2000) and has been extensively studied due to its association with suicide, self-harm, violence, and substance misuse (American Psychiatric Association, 2013). Symptoms of BPD result in high levels of service usage (Bender et al., 2001; Comtois et al., 2003) and high mortality rates (American Psychiatric Association, 2001). Several characteristics of the disorder (e.g. impulsivity, recurrent suicidal behaviour) unfortunately lend themselves to early disengagement from treatment and difficulty committing to and engaging with the therapeutic process. Additionally, BPD is characterised by difficulties in establishing trusting and collaborative interpersonal relationships and, "frantic efforts to avoid real or imagined abandonment" (American Psychiatric Association, 2013), which naturally extend to difficulties in the therapeutic relationship. Indeed, a recent qualitative study confirmed patients' reluctance to be open and honest with their therapist because of fears of rejection and abandonment (Morris et al., 2014). Owing to the stigma associated with BPD, clinicians may find it difficult to communicate the diagnosis in a patient-centred manner (Sulzer et al., 2015), further exacerbating problematic therapist-patient relationships. Although research has sought to identify effective therapeutic treatments for the condition, the majority of BPD research to date focuses on outcome data with relatively few studies identifying reasons why therapies are successful, and what the specific processes through which improvements occur might be (Lynch et al., 2006). Linehan (2000) notes the need to identify 'active' components of psychological therapy so that those aspects can be emphasised when striving for the most effective treatment.

Purpose. To carry out an approach of the current state of behavioral dialectic therapies focused on borderline personality disorder.

Methodology. Searching, identifying and selecting studies for inclusion.

Searches of paper titles, abstracts and full text content were initially performed in July and August 2012 then updated in February 2014, in PsychInfo, PubMed and MEDLINE databases. Search terms used were:

a) "Borderline personality disorder".

b) "approach and mechanism".

c) "DBT therapy".

Studies included in the review involved participants who:

- Met standardised diagnostic criteria for BPD.

- Had received either CBT or DBT treatment for their BPD.

- Were treated as outpatients (due to the limited number of manualised DBT/CBT studies of inpatients or partially hospitalised patients with BPD).

- Were adults (aged 18+ years) at the time of treatment (as there is a limited research presence investigating emerging BPD in adolescents).

Results. Axelrod et al. (2011) posited that greater control of emotions in BPD would lead to less impulsive behaviour which would, in turn, reduce the need to self-medicate using substances to regulate emotions. Females with substance dependence and BPD received a 20 week course of outpatient DBT and emotion regulation was assessed using the Difficulties in Emotion Regulation Scale (Gratz \& Roemer, 2004). Substance use was recorded for 30 days preceding treatment and for the final 30 days of treatment, corroborated by weekly self-report, clinician assessment, urine toxicology and alcohol breathalysers. The study concluded that improvements in 
emotion regulation explained the variance in decreased substance use frequency. Changes in substance use lost their significance when improvement in emotion regulation was controlled for.

Limitations and strengths of the study. In the documentation systems used we have found a limited presence of research that addresses DBT in adolescents. Despite of that, there is a varied therapeutic application.

Impact. Recent research shows that DBT has a positive impact on the reduction of time in treatment, for example, reduction in suicide ideation, depression or episodes of binge eating or purging behaviors associated with eating disorders.

Practical and Social Value. The social and practical value of DBT is evident in the therapies which it is used such as Dialectical Behavior Therapy for Bulimia Nervosa, Treatment of antisocial behavior, Treatment of substance dependency in individual with BPD, etc.

Keywords: Borderline Personality Disorder, CBT, DBT, approach, therapeutic change.

\section{References.}

Addis, M \& Linehan, M.M. (1989). Predicting suicidal behaviour: psychometric properties of the Suicidal Behaviours Questionnaire. Poster presented at: Annual Meeting of the Association for the Advancement Behaviour Therapy; November 2-5, 1989; Washington, DC.

American Psychiatric Association (2013). Diagnostic and Statistical Manual of Mental Disorders (5th Edition). Arlington, VA: American Psychiatric Publishing.

Axelrod, S. R., Perepletchikova, F., Holtzman, K. \& Sinha, R. (2011). Emotion regulation and substance use frequency in women with substance dependence and borderline personality disorder receiving dialectical behaviour therapy. The American Journal of Drug and Alcohol Abuse, 37, 37-42.

Arntz, A. (1994). Treatment of borderline personality disorder: A challenge for cognitive behavioural therapy. Behaviour, Research and Therapy, 32, 419-430.

Wenzel, A., Chapman, J. E., Newman, C. F., Beck, A. T. \& Brown, G. K. (2006) Hypothesised mechanisms of change in cognitive therapy for borderline personality disorder. Journal of Clinical Psychology, 62, 503-516 